\title{
A urbanização das leishmanioses e a baixa resolutividade diagnóstica em municípios da Região Metropolitana de Belo Horizonte
}

\author{
Leishmaniasis urbanization and low diagnosis capacity in the \\ Metropolitan Region of Belo Horizonte
}

\author{
Zélia Maria Profeta da Luz ${ }^{1,2}$, Denise Nacif Pimenta1', Ana Lúcia Lobo Vianna Cabral ${ }^{3}$, \\ Vanessa Oliveira Pires Fiúza ${ }^{3}$ e Ana Rabello ${ }^{1}$
}

\begin{abstract}
Resumo No período de 1994 a 1999, foram notificados casos de leishmaniose tegumentar em 32 (89\%) dos 36 municípios da Região Metropolitana de Belo Horizonte. Em um (2,8\%) município o risco de adquirir a doença foi considerado muito alto, em 16 (44.5\%), médio em sete (19,4\%) e baixo em 12 (33.3\%). Leishmaniose visceral foi notificada em seis (17\%) dos 36 municípios, nos anos 94 e 95, elevando-se para 15 (42\%) no biênio 98/99. O total de casos de leishmaniose visceral notificados anualmente no período 94 a 99 foi 30, 53, 64, 60, 53, 84, respectivamente. Não há serviços referenciados para atendimento da doença em $19(61,3 \%)$ de 31 municípios, sendo $80 \%$ dos pacientes encaminhados para Belo Horizonte. Em 12 (39\%) municípios com serviços referenciados, somente oito (67\%) dispõem de testes diagnósticos específicos para leishmaniose. Verificouse rápida e extensa expansão das leishmanioses na região metropolitana de Belo Horizonte e baixa capacidade de resolução diagnóstica pelos seus municípios.
\end{abstract}

Palavras-chaves: Leishmanioses. Diagnóstico. Municípios. Região Metropolitana de Belo Horizonte. Serviços de saúde.

\begin{abstract}
During the period from 1994 to 1999 cutaneous leishmaniasis was reported in 32 (89\%) out of 36 municipalities in the Metropolitan Region of Belo Horizonte, Brazil, of which one $(2,8 \%)$ municipality was classified as a very high risk area, 16 (44,5\%) as high risk, seven (19,4\%) as moderate risk areas and $12(33,3 \%)$ as low risk. From 1994 to 1995, visceral leishmaniasis was reported in six (16\%) municipalities whereas in 1998 1999 this number increased to 15 (42\%). Annual numbers of cases during 1994 to 1999 were 30, 53, 64, 53 and 84, respectively. In 19 (61.3\%) municipalities no reference center for the diagnosis of the infection was available, so that most of the patients (80\%) were referred to Belo Horizonte. Twelve (39\%) municipalities have a center for leishmaniasis evaluation, however in only eight (67\%) of these basic specific diagnostic tests were available. Rapid and extensive increase of leishmaniasis associated with low diagnosis capacity has been observed in the metropolitan area of Belo Horizonte.
\end{abstract}

Key-words: Leishmaniasis. Diagnosis. Municipalities. Metropolitan Region of Belo Horizonte. Health care.

As leishmanioses constituem grave problema de saúde pública no Brasil com a leishmaniose tegumentar americana (LTA) sendo diagnosticada em praticamente todos os estados brasileiros. Houve redução dessa forma clínica na década de 50, mas nos últimos 20 anos o número de casos notificados vem aumentando progressivamente, com média anual de 28.000 casos nos últimos dez anos ${ }^{7}$. A leishmaniose visceral americana (LVA), tem sido notificada em 17 estados, atingindo quatro das cinco regiões geográficas do país. Sua maior incidência encontra-se no Nordeste, com $92 \%$ do total das notificações, seguida pelas regiões Sudeste $(4 \%)$, Norte (3\%) e Centro-Oeste $(1 \%)^{4}$.
Em Minas Gerais, no período de 1988 a 1999, a média anual do número de casos notificados de LTA foi de 1.910 e de LVA, 127. Nesse mesmo período, verificou-se aumento expressivo de municípios com registro de leishmaniose. Em 1999, a LTA foi notificada em 400 municípios e a LVA em 47, representando ambos, o dobro de municípios com a doença, se comparado a 1988 (SES/MG: dados não publicados). Na Região Metropolitana de Belo Horizonte (RMBH), com população residente predominantemente urbana, casos autóctones de LTA L LVA têm sido registrados pelos órgãos oficiais de saúde desde 1987 e 1989, respectivamente ${ }^{85}$. Neste estudo, avaliou-se a distribuição e a capacidade de resolução diagnóstica das leishmanioses nos 36 municípios da RMBH.

\footnotetext{
1. Laboratório de Pesquisas Clínicas, Centro de Pesquisas René Rachou/Fundação Oswaldo Cruz. 2. Departamento de Parasitologia da Universidade Federal de Minas Gerais. 3. Diretoria Metropolitana de Saúde da Secretaria Estadual de Saúde de Minas Gerais, Belo Horizonte, MG.

Apoio financeiro: Fundação Oswaldo Cruz e CNPq

Endereço para correspondência: Dra. Zélia Maria Profeta da Luz.Centro de Pesquisas René Rachou/FIOCRUZ, Av. Augusto de Lima 1715, Barro Preto, 30190-002 Belo Horizonte, MG

Tel: 55313295 3566, Fax: 553132953115

e-mail:profeta@cpqrr.fiocruz.br

Recebido para publicação em 03/7/2000.
} 


\section{MATERIAL E MÉTODOS}

Caracterização dos municípios em função do nível de atenção dos serviços de saúde. A RMBH incluindo a capital é composta por 36 municípios, com população total de 4.293 .433 habitantes (http:// www.datasus.gov.br). Na Figura 1, está representado o Estado de Minas Gerais, com os municípios da RMBH, suas respectivas populações e níveis de atenção dos serviços de saúde. Foram considerados municípios da $\mathrm{RMBH}$ aqueles pertencentes à área de abrangência da Diretoria Metropolitana de Saúde da Secretaria de
Estado da Saúde de Minas Gerais (DMS-SES). Os municípios com nível de atenção primária dispõem apenas de unidades básicas de atendimento; os de nível secundário possuem unidades básicas e unidades de atendimento 24 horas e os de nível terciário contam com unidades básicas, unidades 24 horas e hospitais. Em 1999, nove (25\%) municípios dispunham de atendimento de nível primário, 19 (53\%) de nível secundário e 8 (22\%) de nível terciário.

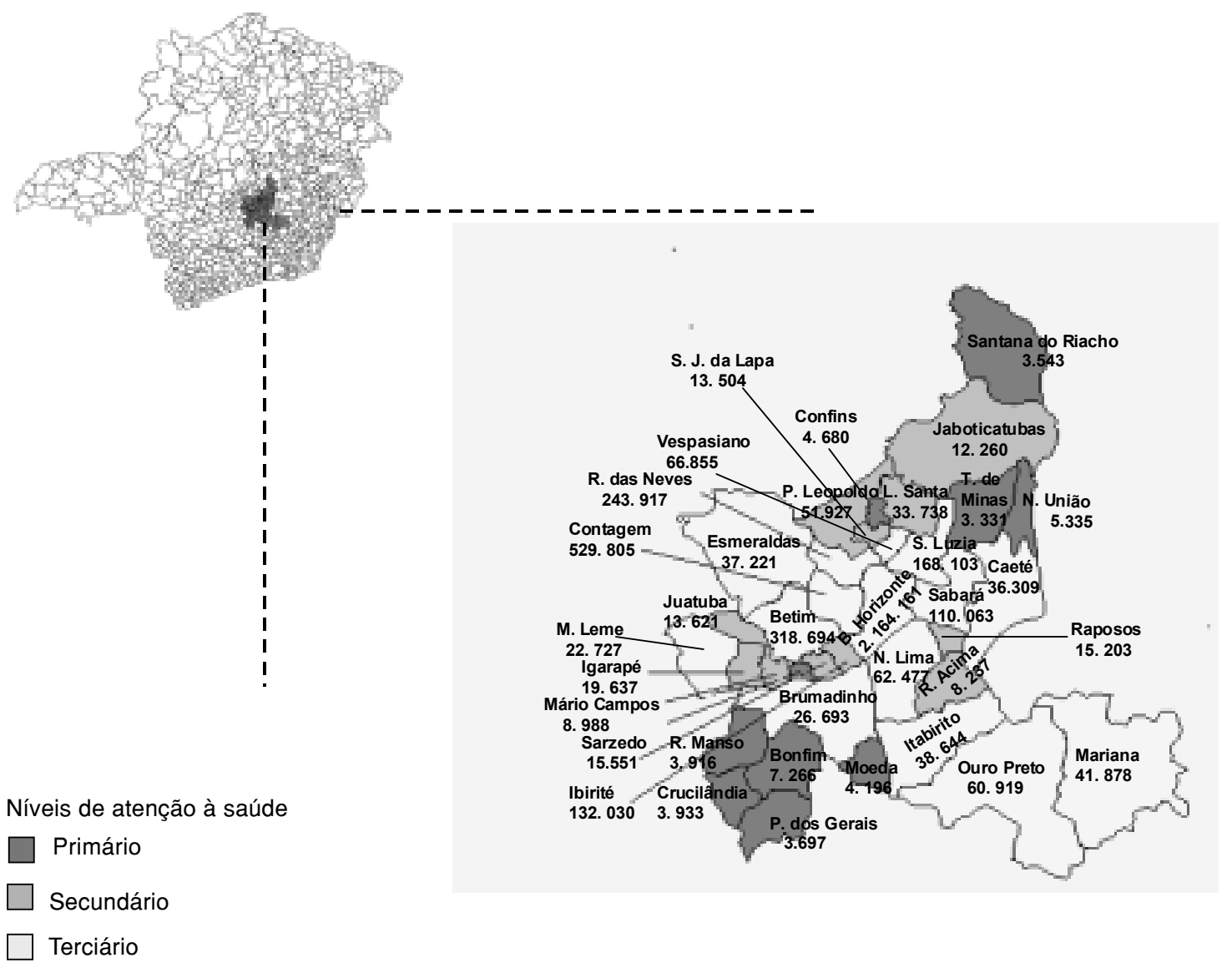

Figura 1 - Mapa de Minas Gerais com destaque para os municípios da Região Metropolitana de Belo Horizonte suas respectivas populações e níveis de atenção dos serviços de saúde.

Incidência e risco de adoecer. A incidência foi calculada em função do número de casos por cem mil habitantes por município de autoctonia no período de 1994 a 1999. O risco de adoecer para LTA $^{3}$ foi baseado na incidência/100.000 hab, sendo considerado risco baixo aquele com incidência menor ou igual a três; risco médio, incidência entre três a 11; risco alto, incidência de 11 a 71 e risco muito alto, incidências maiores que 71.
Infra-estrutura para atendimento dos casos de leishmanioses. A existência de serviços referenciados e a disponibilidade de estrutura física e de recursos humanos para o atendimento às leishmanioses em 1999 foram analisados através de questionários enviados aos municípios. Excluiu-se Belo Horizonte, devido à complexidade de estrutura e serviços neste município. 


\section{RESULTADOS}

Caracterização dos municípios em relação à distribuição da LTA. Até o momento, casos de LTA foram notificados em 32 (89\%) municípios da RMBH. O número de casos notificados de LTA na RMBH, no período de 1994 a 1999 está representado na Figura $2 \mathrm{~A}$. Houve redução desses números nos anos 95 a 97 em relação a 94 , porém, aumento acentuado de LTA

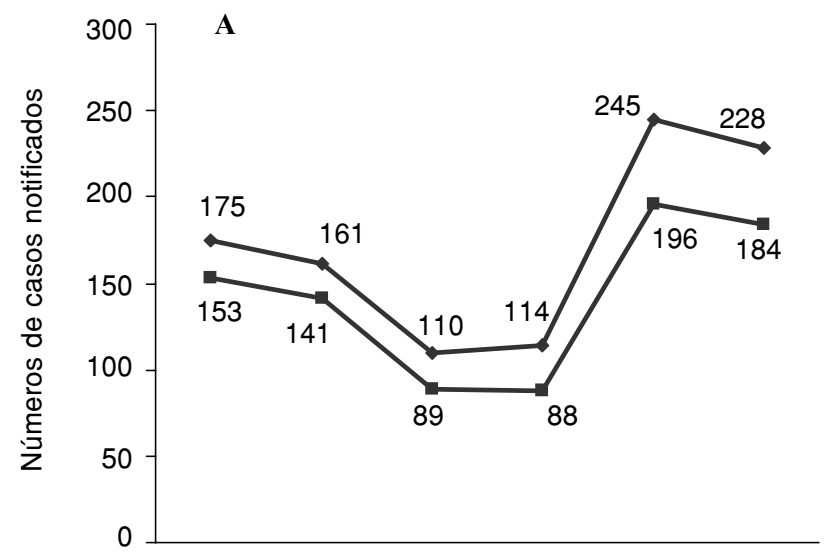

- RMBH com Belo Horizonte

- RMBH sem Belo Horizonte

Figura 2 A - Distribuição de casos de LTA.

A incidência da LTA em função do nível de atenção nos diferentes anos encontra-se na Tabela 1. No período 94-95, foram notificados casos de LTA em três dos dez municípios com nível de atenção primário e nos dois últimos anos, em cinco. Nos municípios com nível secundário de atenção, verificou-se redução da ocorreu em 98 e 99. Em geral, o risco de adquirir a doença, demonstrado na Figura $2 \mathrm{~B}$, foi constante no período. Do total de 36 municípios verificou-se 19 com risco baixo de adoecer no ano de 97 e 18 municípios com risco alto em 98 . Risco muito alto de adquirir a LTA foi observado em um município (Juatuba).

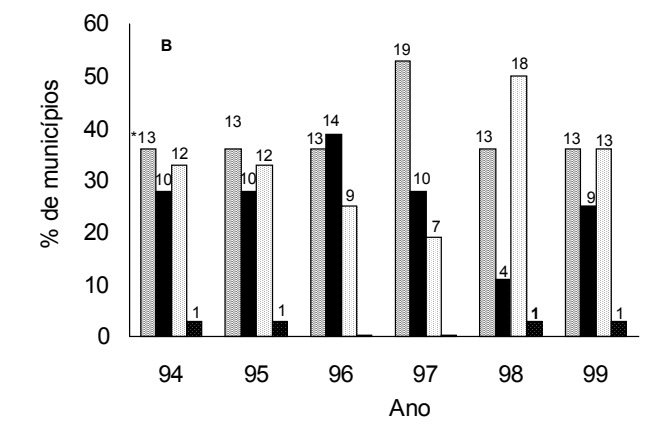

*número de municípios

$$
\square \text { Baixo: } \leq 3 \square \text { Médio: } 3 \text { a } 11 \square \text { Alto: } 11 \text { a } 71 \square \text { Muito alto: } \geq 71
$$

Figura $2 B$ - Risco de adquirir a doença em 36 municípios da $R M B H$ no período de 1994 a 1999.

incidência nos anos 96-97. No último quadriênio foram detectados casos da LTA em $100 \%$ dos municípios. A incidência manteve-se constante no período de 94 a 99 nos municípios com atendimento terciário.

Caracterização dos municípios em relação à distribuição da LVA. Em 15 (42\%) dos 36 municípios

Tabela 1- Incidência média de LTA, no período de 1994 a 1999, em 36 municípios da RMBH/DMS-SES, com diferentes níveis de atenção.

\begin{tabular}{lcccc}
\hline Nível de atenção & $\begin{array}{c}\text { Período } \\
\text { (ano) }\end{array}$ & $\begin{array}{c}\text { Municípios com LTA } \\
\text { total de municípios }\end{array}$ & $\begin{array}{c}\text { Incidência média } \\
\text { (mínimo-máximo) }\end{array}$ & $\begin{array}{c}\text { Pop.média } \\
\text { (mínimo-máximo) }\end{array}$ \\
\hline \multirow{3}{*}{ Primário } & $94-95$ & $3 / 10$ & $31,9(0-43,37)$ \\
& $96-97$ & $1 / 10$ & $42,73(0-56,53)$ & $34,61(0-54,8)$ \\
\hline \multirow{3}{*}{ Secundário } & $98-99$ & $5 / 10$ & $33,85(4,06-131,36)$ \\
& $94-95$ & $10 / 11$ & $14,46(3,02-52,42)$ & $28.537(7.476-125.982)$ \\
\hline \multirow{3}{*}{ Terciário } & $96-97$ & $11 / 11$ & $38,14(3,03-208,71)$ & $1(3.369-8.856)$ \\
& $98-99$ & $11 / 11$ & $9,04(0,81-56,53)$ & $129.779(20774-2.091 .371)$ \\
\hline
\end{tabular}

LTA: leishmaniose tegumentar americana; RMBH/DMS-SES: Região Metropolitana de Belo Horizonte/ Diretoria Metropolitana de Saúde da Secretaria Estadual de Saúde 
houve notificação de LVA no período $94 / 99$, sendo o número total de casos $30,53,64,60,53,84$, respectivamente (Figura 3). Chama atenção que, excluindo-se Belo Horizonte, verifica-se aumento da doença, sendo os números no mesmo período $1,7,16$, 13, 29, 53 (Figura 3). Em 12 (80\%) desses municípios, o atendimento era terciário e em três (20\%) verificou-

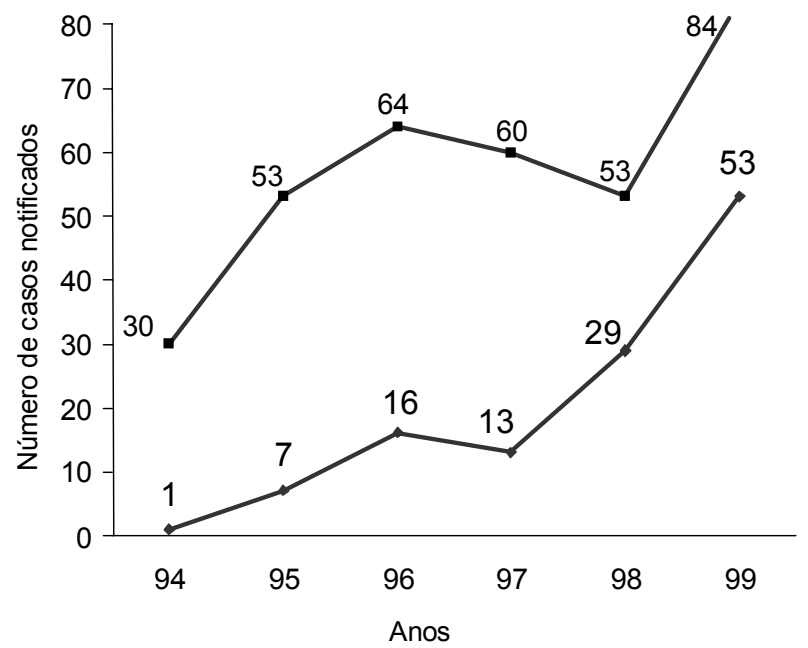

- $\mathrm{RMBH}$ incluindo Belo Horizonte

- RMBH excluindo Belo Horizonte

Figura 3 - Total de casos de leishmaniose visceral notificados em 15 dos 36 municipios da RMBH.

Caracterização dos municípios quanto à infraestrutura dos serviços de saúde. Dos 36 questionários enviados às secretarias de saúde dos municípios, foram respondidos 31 (86\%). Não existem serviços de referência em 19 (61\%), sendo os pacientes encaminhados para outros municípios, $80 \%$ para Belo Horizonte, com distância média de $30 \mathrm{~km}$, variando de 17 até $96 \mathrm{~km}$. Nos 12 municípios onde existem serviços referenciados, oito (67\%) dispõem de recursos diagnósticos. Nesses casos, a intradermorreação de Montenegro (IDRM) foi o método diagnóstico disponível em $75 \%$. A freqüência de interesse em implantar ou aperfeiçoar o diagnóstico no próprio se níveis de atenção secundário. O total de municípios com notificação de LVA elevou-se de 6 em 94/95 para 15 nos anos 98/99 (Figura 4). A incidência foi variável, com valores elevados nos dois últimos anos. Verificouse redução da incidência em apenas dois municípios, sendo que em um deles, casos não têm sido registrados desde 1997 (Figura 4).
Incidência/100.000

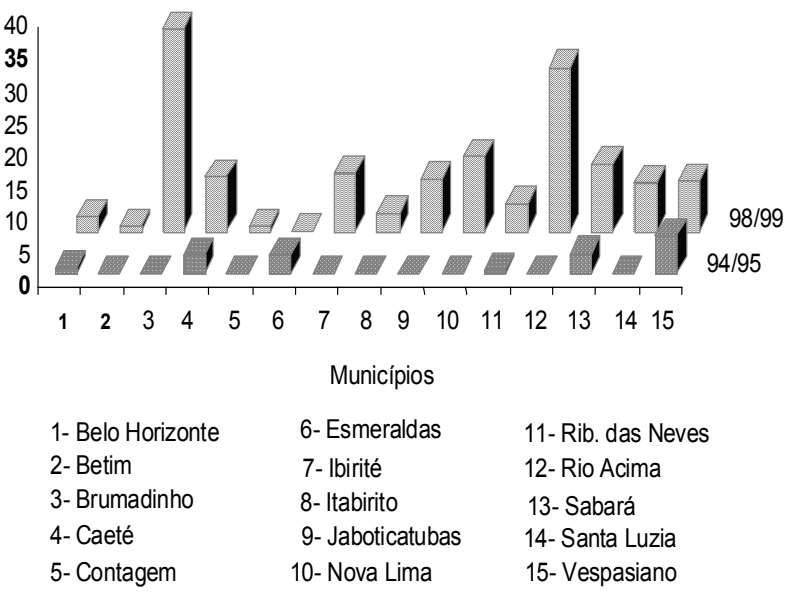

Figura 4 - Incidência da leishmaniose visceral em 15 dos 36 municípios da da RMBH, nos biênios 94/95 e 98/99.

município está demonstrado na Figura 5. Houve igual interesse em implantar IDRM, sorologia e identificação do parasito. Estrutura física para a realização de algum método diagnóstico foi registrada em 14 (45\%) municípios. Com relação à existência de recursos humanos disponíveis para o treinamento, estes foram detectados em 24 (77\%) municípios sendo 17 (55\%) profissionais de nível superior e os demais de nível médio. Em três (10\%), não há interesse na realização do diagnóstico no próprio local. Os principais motivos citados foram a falta de estrutura física e/ou de recursos humanos ou a ausência de casos notificados

\section{DISCUSSÃO}

A expansão das leishmanioses em Minas Gerais fica evidente quando se avalia o número de municípios com casos notificados nos últimos anos. Esse foi o dobro em 1999 quando comparado ao ano 1988 (SES/MG: dados não publicados).

A caracterização da ocorrência de transmissão das leishmanioses na RMBH como urbana se deve ao crescente número de casos nesta região, onde a população urbana é predominante. Em Belo Horizonte, município que concentra o maior número de casos, não há área rural. No ano de 1994, 29 das 30 notificações na $\mathrm{RMBH}$ eram provenientes de Belo Horizonte. Registro significativo da doença também foi observado em Contagem, Nova Lima, Ibirité, Santa Luzia, 

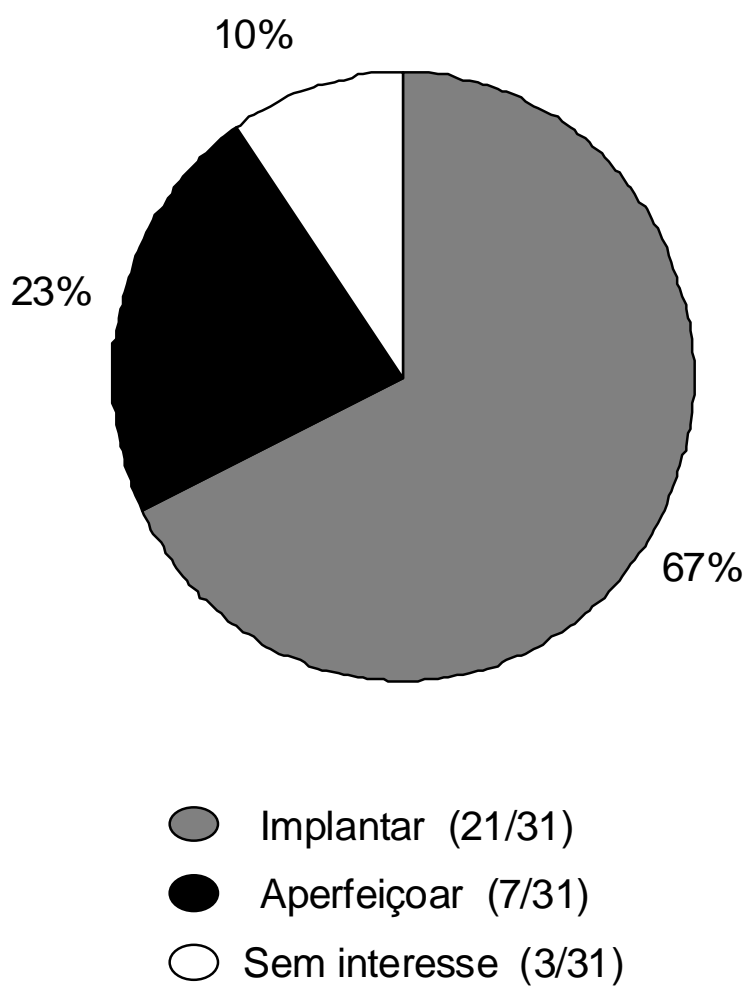

Figura 5 - Percentual de 31 municípios da $R M B H$, com interesse em implantar ou aperfeiçoar, no próprio município, metodologias para o diagnóstico laboratorial da lesihmaniose.

Vespasiano, Juatuba, Sabará, Ribeirão das Neves, com 96,7\% a 99,5\% da população vivendo em áreas urbanas (http://www.sidra.ibge.gov.br). Além disso, análise parcial de 168 fichas individuais de notificação de LV mostra que $91,4 \%$ dos casos autóctones da $\mathrm{RMBH}$ residiam em áreas urbanas.

A avaliação da LTA, no período de 1980 a 1991, mostrou que Minas Gerais foi o estado com o maior número de casos em toda a Região Sudeste ${ }^{7}$. Das 22 primeiras notificações da LTA na RMBH em 1987, dez eram autóctones, sendo três de Belo Horizonte. Os outros sete casos foram registrados nos municípios de Sabará, Rio Acima e Raposos ${ }^{8}$. Passos e cols ${ }^{10}$ verificaram numa casuística composta de 388 pacientes, uma nova situação epidemiológica no estado, com expansão da doença a regiões até então consideradas indenes. Esses mesmos autores verificaram após inquérito populacional realizado em área periurbana de Sabará, a ocorrência da LTA em ambos os sexos e em crianças, sugerindo transmissão peridomiciliar. Além disso, foram encontradas espécies de flebotomíneos em áreas de mata, no peridomicílio e no intradomicílio ${ }^{9}$. Em 1999, a LTA já atinge, 32 (89\%) dos 36 municípios da $\mathrm{RMBH}$. O risco de adoecer foi baixo em apenas 13 $(36 \%)$ destes municípios. Nos demais, esse risco foi médio, alto ou muito alto, o que torna a situação preocupante, considerando a expansão da doença, aliada às mudanças no perfil de transmissão.

Observou-se predomínio do risco baixo de adoecer no ano de 97. Como o fornecimento de antimonial pentavalente, utilizado no tratamento da leishmaniose, é condicionado à entrega da ficha de notificação, o predomínio do risco baixo em 97 pode ser devido à descontinuidade do fornecimento do medicamento, ocorrido naquele ano.

A maior ocorrência de surtos urbanos de leishmaniose, quando comparada a outras parasitoses, pode ser explicada por sua capacidade de expansão de forma muito rápida quando introduzida em área não endêmica ${ }^{12}$. De fato, com relação à LVA, o percentual de municípios com notificação elevou-se de 6, no biênio 94/95, para 15 no biênio 98/99, mostrando claramente rápida expansão da doença na RMBH. Chama a atenção o número de casos de LVA, no ano 99 que foi 53 vezes maior em relação a 94 quando excluiu-se Belo Horizonte. A LVA acarreta elevado custo social, medido em anos potenciais de vida perdidos, devido a maior ocorrência de óbitos em grupos etários mais jovens ${ }^{11}$. No Estado de Minas Gerais foram registrados 85 óbitos devido à leishmaniose entre 1990 a 1997 (http://www.datasus.gov.br). Desses, 21\% 
ocorreram na RMBH, com número de óbitos no período 94/97, 2,6 vezes maior que o ocorrido entre 1990 a 1993. Além disso, em geral, são precárias as condições sócio-econômicas dessas populações atingidas, agravando o problema.

Outro ponto que merece destaque é que provavelmente o número de casos diagnosticados de LVA representa apenas uma pequena fração das pessoas infectadas pela $L$. chagasi ${ }^{6}$. Em estudos prospectivos feitos na Bahia e Ceará, a taxa de indivíduos assintomáticos/sintomáticos era respectivamente $6: 1$ a 18:1, dependendo da idade e da localidade estudada ${ }^{12}$.

A inexistência na RMBH de estudos sistematizados enfocando vetores, reservatórios, e modificações do ambiente dificultam a análise de fatores determinantes da expansão das leishmanioses nessa região.

O aumento do número de casos de leishmanioses na $\mathrm{RMBH}$ poderia ser resultado da melhoria do diagnóstico realizado nos municípios. Entretanto, verificou-se que não existem na RMBH serviços de referência para esse diagnóstico em pelo menos $60 \%$ dos municípios. Assim, a maioria (80\%) dos pacientes tem que ser encaminhada para Belo Horizonte.

Estes dados evidenciam a rápida e extensa distribuição das leishmanioses na RMBH e a grave deficiência de capacitação e de infraestrutura necessárias para a abordagem adequada da infecção.

\section{REFERÊNCIAS BIBLIOGRÁFICAS}

1. Badaró R, Jones TC, Lorenço R, Cerf BJ, Sampaio D, Carvalho EM, Rocha H, Teixeira R, Jonhson WD. A prospective study of visceral leishmaniasis in an endemic area of Brazil. Journal of Infectious Diseases 154: 639-649, 1986.

2. Evans TG, Teixeira MJ, McAulife IT, Vasconcelos I, Vasconcelos AW, Sousa AD, Lima JW, Pearson RD. Epidemiology of visceral leishmaniasis in northeast Brazil. Journal of Infectious Diseases 166: 1124-1132, 1992.

3. Fundação Nacional de Saúde. Leishmaniose Tegumentar Americana (LTA). In: Guia de Vigilância Epidemiológica. Ministério da Saúde 4 edição, Brasília, 1998.

4. Fundação Nacional de Saúde. Leishmaniose visceral (LVA). In: Guia de Vigilância Epidemiológica. Ministério da Saúde, 4 edição, Brasília, 1998.

5. Genaro O, Costa CA, Williams P, Silva JE, Rocha NM, Lima SL, Mayrink W. Ocorrência de calazar em área urbana da Grande Belo Horizonte. Revista da Sociedade Brasileira de Medicina Tropical 23: 121, 1990.

6. Jeronimo SMB, Oliveira RM, Mackay S, Costa RM, Sweet J, Nascimento ET, Luz KG, Fernandes MZ, Jernigan J, Pearson RD. An urban outbreak of visceral leishmaniasis in Natal, Brazil. Transactions of Royal Society of Tropical Medicine and Hygiene 88: 386-388, 1994.

7. Lacerda MM. The Brazilian leishmaniasis Control Program. Memórias do Instituto Oswaldo Cruz 89: 489-495, 1994.

8. Passos VMA, Andrade AC, Silva ES, Figueiredo EM, Falcão AL. Inquérito canino em foco recente de leishmaniose tegumentar no município de Sabará, região metropolitana de Belo Horizonte. Revista da Sociedade Brasileira de Medicina Tropical 29: 323 329, 1996.

9. Passos VMA, Falcão AL, Katz N. Urban american cutaneous leishmaniasis in the metropolitan region of Belo Horizonte, Minas Gerais state, Brazil. Memórias do Instituto Oswaldo Cruz 85: 243244, 1990.

10. Passos VMA, Falcão AL, Marzochi MCA, Gontijo CMF, Dias ES, Barbosa-Santos EGO, Guerra HL, Katz N. Epidemiological aspects of american cutaneous leishmaniasis in a periurban area of the metropolitan region of Belo Horizonte, Minas Gerais, Brazil. Memórias do Instituto Oswaldo Cruz 88: 103-110, 1993.

11. Vieira FJB, Coelho GE. Leishmaniose visceral ou calazar: aspectos epidemiológicos e de controle. Revista da Sociedade Brasileira de Medicina Tropical 31 (supl II): 85-92, 1998.

12. Wijeyaratne PM, Arsenault J, Murphy CJ. Endemic disease and development: the leishmaniasis. Acta Tropica 56 : 349364, 1994. 